\title{
Odourless Watermark (Digital Chemocode) System with Biochemical Sniff Scanner
}

\author{
Hirokazu Saito, Teruyoshi Goto ${ }^{1}$, Kumiko Miyajima1, \\ Munkhbayar Munkhjargal ${ }^{1}$, Takahiro Arakawa ${ }^{1}$ and Kohji Mitsubayashi ${ }^{1, *}$ \\ Department of Mechanical Engineering, Tokyo National College of Technology, \\ 1220-2 Kunugida, Hachioji, Tokyo 193-0997, Japan \\ ${ }^{1}$ Department of Biomedical Devices and Instrumentation, Institute of Biomaterials and \\ Bioengineering, Tokyo Medical and Dental University, \\ 2-3-10 Kanda-Surugadai, Chiyoda-ku, Tokyo 101-0062, Japan
}

(Received September 26, 2013; accepted November 20, 2013)

Key words: $\quad$ watermark, biosniffer, odourless, volatile chemical, enzyme

Many types of identification systems such as watermark, barcode, integrated circuit (IC) tag, and fingerprint systems have been developed and utilized. These identification systems recognize physical information of the identification medium for certification information. However, an identification system that detects the chemical characteristics of a subject is not yet practicable. In this study, an odourless and invisible watermark system was developed using biochemical gas sensors (biosniffers) for detecting encoded chemical information. Each biosniffer consisted of a Clark-type dissolved oxygen electrode and an enzyme-immobilized membrane. Each enzyme (catalase, lactate oxidase, or choline oxidase) was immobilized onto a dialysis membrane by photocrosslinking with polyvinyl alcohol containing stilbazolium groups. The calibration ranges of the biosniffers for hydrogen peroxide, lactic acid, and choline vapours were from 0.4 to $12.5,0.01$ to 10.0 , and 1.0 to $1000 \mathrm{ppm}$ and the correlation coefficients were $0.996,0.975$, and 0.956 , respectively. Each biosniffer showed a linear response to the concentration of the substrate in the gas phase. These biosniffers were used for scanning 3 bits (eight patterns) of the digital chemocode made of hydrogen peroxide, lactic acid, and choline solutions on filter paper. The three types of biosniffer successfully recognized eight patterns of odourless chemical codes.

\section{Introduction}

Watermarks, barcodes, integrated circuit (IC) tags, biometrics (finger/palm print, finger/palm vein, iris), and other such information have been applied for authentication systems that recognize the physical characteristics of visible substances. ${ }^{(1-5)}$ However,

${ }^{*}$ Corresponding author: e-mail: m.bdi@tmd.ac.jp 
an authentication system that detects the chemical characteristics of a subject has not been practically utilized. In our environment, there are many odourless and invisible substances such as hydrogen peroxide. If the presence/absence of odourless and colourless volatile chemicals can be coded as digital information (digital chemocode), a high-level security system with latent information code can be achieved. For example, if three types of volatile chemical are coded, then 3 bits, namely, 8 patterns of digital chemocodes can be assigned.

As odourless and colourless volatile chemicals for the digital chemocode, hydrogen peroxide, lactic acid, and choline can be cited. Hydrogen peroxide is a strong oxidizing agent and has strong antimicrobial activity. Therefore, hydrogen peroxide is often used for the disinfection of foodstuff, medical or biological equipment and for bleaching textiles, paper pulp, leather, hair, and teeth. ${ }^{(6,7)}$ A low concentration of hydrogen peroxide is also detected in exhaled breath, and its concentration is increased in patients with inflammatory respiratory diseases such as asthma, chronic obstructive pulmonary disease, and cystic fibrosis..$^{(8-13)}$ The American Conference of Governmental Industrial Hygienists (ACGIH) has assigned hydrogen peroxide a threshold limit of $1 \mathrm{ppm}$ as a time-weighted average (TWA) for a normal 8-h workday and a 40-h workweek. ${ }^{(14)}$ Therefore, a low concentration of gaseous hydrogen peroxide (less than $1 \mathrm{ppm}$ ) will be available for the digital chemocode without biological influence. Lactic acid is known as a chemical compound that plays a role in several biochemical processes. In animals, L-lactate is constantly produced from pyruvate during normal metabolism and exercise. Choline is widely distributed in foodstuff in the form of free choline and phosphatidylcholine; it is well known as an important substance for neural transmission in the brain and related with nervous symptoms such as anxiousness, excitation, confusion, and coma. Therefore, amperometric microsensors for choline were reported in the field of brain science. ${ }^{(15,16)}$

To detect the coded low concentration of volatile chemicals, highly sensitive gas sensors are required. In addition, the gas sensors should have gas selectivities to each bit of the volatile chemical and not respond to other chemicals (other bits) in a usage environment. For example, in the standard method of measuring atmospheric hydrogen peroxide vapour concentration, the concentration is measured colorimetrically. ${ }^{(17)}$ However, the colorimetric measurement requires a complicated procedure and expensive and large equipment. As a conventional method for the measurement of gaseous chemical substances, a gas detection tube is generally used. The detector tube allows for a very convenient measurement; however, frequent measurements can be costly. Several sensors for chemical substance vapours have been developed with semiconductors ${ }^{(18)}$ or Nafion ${ }^{\circledR}$ membrane. ${ }^{(19,20)}$ Sensors with semiconductors tend to show low selectivity, and thus are inadequate for sensing multianalyte samples such as expiratory gas or industrial gas. ${ }^{(21)}$ On the other hand, many types of biosensor with enzymes and antibodies for ethanol, acetaldehyde, methyl mercaptan, and trimethylamine have been constructed.(22-24) Owing to the substrate specificity of enzymes and other biomaterials, these sensors show high selectivity. Several biosensors for hydrogen peroxide, ${ }^{(25-28)}$ lactic acid, ${ }^{(29-34)}$ and choline $^{(35-40)}$ have been fabricated and showed good selectivity. Moreover, in recent years, biosensors have also been applied to the detection of gaseous substances. ${ }^{(41-44)}$ 
In this study, we developed an odourless watermark system consisting of biochemical gas sensors (biosniffers) for hydrogen peroxide, lactic acid, and choline where the decomposition of the chemical substances is by the following reactions:

$$
\begin{gathered}
\text { catalase } \\
\text { hydrogen peroxide } \rightarrow 2 \mathrm{H}_{2} \mathrm{O}+\mathrm{O}_{2} \\
\text { lacate oxidase } \\
\text { L-lactic acid }+\mathrm{O}_{2} \rightarrow \text { pyruvate }+\mathrm{H}_{2} \mathrm{O}_{2} \\
\text { choline oxidase } \\
\text { choline }+\mathrm{O}_{2}+\mathrm{H}_{2} \mathrm{O} \rightarrow \text { betaine }+\mathrm{H}_{2} \mathrm{O}_{2}
\end{gathered}
$$

which were catalyzed by catalase, lactate oxidase (LOD), and choline oxidase (COD). Changes in the dissolved oxygen concentration were detected using an oxygen electrode. The responses, calibration properties, and selectivities of these sensors in the gas phase were evaluated. These biosniffers were then used for scanning 3 bits of the digital chemocodes as an odourless watermark system.

\section{Experimental Section}

\subsection{Materials}

An odourless watermark system was constructed using biosniffers, a personal computer (PCG-FX11V, SONY, Tokyo, Japan), a computer-controlled potentiostat (Model 1112, FUSO Inc, Kawasaki, Japan), and an analog-to-digital converter (ADC-16, Pico Technology Ltd., St Neots, UK).

The biosniffers were constructed using commercially available Clark-type dissolved oxygen electrodes (Model BO-P, ABLE Co., Tokyo, Japan) and enzyme membranes. The enzyme membranes were prepared from catalase (EC1.11.1.6: from bovine liver, 5000 - $13000 \mathrm{U} \mathrm{mg}^{-1}$, No. 039-12901, Wako Pure Chemical Industries, Ltd., Tokyo, Japan), lactate oxidase (LOD, EC 1.1.3.2: from Pediococcus sp, $47 \mathrm{U} \mathrm{mg}^{-1}$, code L-638, Sigma-Aldrich Japan K.K., Tokyo, Japan), choline oxidase (COD, E.C. 1.1.3.17: from Alcaligenes sp., $10 \mathrm{U} \mathrm{mg}^{-1}$, T-0043, Funakoshi Co. Ltd., Tokyo, Japan), photocrosslinkable polyvinyl alcohol containing stilbazolium groups [PVA-SbQ: Type: Styryl Pyridinium polymer-H-13 (Bio), Toyo Gosei Co. Ltd., Tokyo, Japan], ${ }^{(45)}$ and an ultrathin dialysis membrane (part no. 157-0144-02, thickness: $15 \mu \mathrm{m}$, Technicon Chemicals Co., S.A., Oecq, Belgium). A qualitative filter paper (No.2, thickness: $0.25 \mathrm{~mm}$, Advantech Toyo Co. Ltd., Tokyo, Japan) was used as a recording paper of digital chemocodes, and a gas-sampling bag $\left(880 \mathrm{ml}\right.$, SAN GIP G-4, $200 \times 140 \times 0.04 \mathrm{~mm}^{3}$, C.I. Sanplus Co. Ltd., Tokyo, Japan) was used for vaporizing hydrogen peroxide. A gas detector tube for hydrogen peroxide vapour (No. 32, Gastec Corp., Kanagawa, Japan) was used to measure the concentration of the hydrogen peroxide vapour. 


\subsection{Construction of biosniffers}

The biosniffers for hydrogen peroxide, lactic acid, and choline vapours were constructed using the Clark-type dissolved oxygen electrode and the catalase-, lactate LOD-, and COD-immobilized membrane, respectively. Figure 1 shows the process of immobilizing the enzymes onto the dialysis membrane and a schematic of the biosniffer. The enzyme was mixed with photocrosslinkable polyvinyl alcohol containing stilbazolium groups (PVA-SbQ, Toyo Gosei Co. Ltd., Tokyo, Japan) in a weight ratio of 1:100, then coated on the dialysis membrane $\left(3.0 \times 9.0 \mathrm{~cm}^{2}\right)$, which was rinsed with distilled water and dried on a plastic plate before spreading of the mixture. The enzymecoated membrane was desiccated in the dark below $10{ }^{\circ} \mathrm{C}$ for $1.0 \mathrm{~h}$ and then irradiated with fluorescent light of a commercially available illumination tube for $30 \mathrm{~min}$ to photocrosslink the PVA-SbQ, immobilizing the enzyme on the dialysis membrane. The enzyme-immobilized membranes were kept at $2{ }^{\circ} \mathrm{C}$ in a refrigerator when not in use. The enzymatic activities of these immobilized enzymes were maintained for over two months.

By using each enzyme-immobilized membrane, the biosniffers for hydrogen peroxides, lactic acid, and choline vapours were fabricated by the following procedure. The enzyme-immobilized membrane was cut into a square of $10 \mathrm{~mm}$ on each side. Then, a drop of the phosphate buffer solution was put on the sensing area of the oxygen electrode, and a piece of the enzyme-immobilized membrane was attached and secured with a supporting nylon mesh and a silicone O-ring. In the preparatory experiments, the effect of $\mathrm{pH}$ on the output of these biosniffers was evaluated. The optimal $\mathrm{pH}$ for catalase and LOD was 7.0, and that for COD was 8.0. Therefore, a drop (20 $\mu 1)$ of

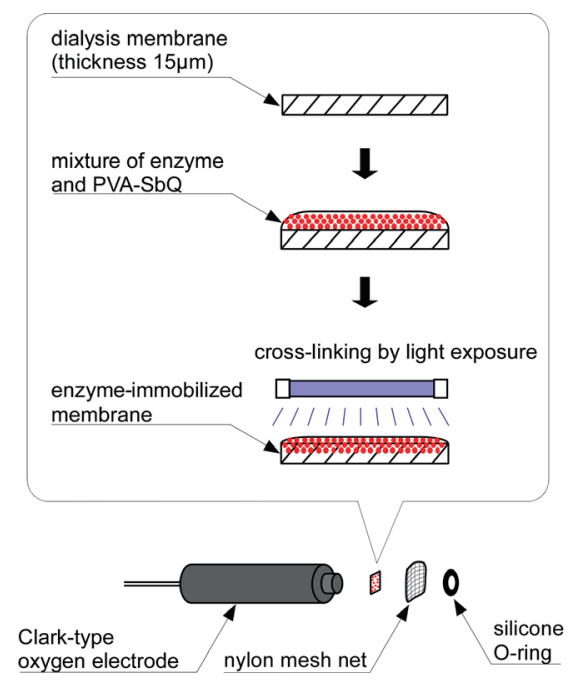

Fig. 1. (Color online) Construction of an enzyme-immobilized membrane and a biosniffer. 
the phosphate buffer solution was put on the sensing area of the biosniffer for enzyme activation. These biosniffers detect the hydrogen peroxide, lactic acid, and choline by monitoring the changes in the oxygen concentration caused by the reaction described in eqs. (1)-(3).

\subsection{Experimental setup for evaluation of biosniffers}

The performances of the biosniffers were assessed in the gaseous phase. The biosniffer device was provided with a fixed potential of $-700 \mathrm{mV}$ versus $\mathrm{Ag} / \mathrm{AgCl}$ as counter/reference electrodes using the potentiostat. This potential was chosen in accordance with the specification of the oxygen electrode. The output current of the biosniffer device was sent to the computer via the analog-to-digital converter. Then, the biosniffer output induced by the enzyme reaction [eqs. (1)-(3)] was continuously monitored on the computer display and stored in the hard disk. All the experiments were carried out at room temperature $\left(\mathrm{ca} .20^{\circ} \mathrm{C}\right)$.

To evaluate the characteristics of the biosniffer response and cross talk between the sensor outputs, hydrogen peroxide, lactic acid, and choline vapours were applied to the biosniffers. $20 \mu \mathrm{l}$ of hydrogen peroxide solution at various concentrations was put on a $20 \times 25 \mathrm{~mm}^{2}$ filter paper and set into the gas sampling bag. The sampling bag was filled with air and kept at room temperature for $20 \mathrm{~min}$. Then, the sensitive area of the biosniffer was inserted into the sampling bag and the change in the sensor output was continuously monitored. The concentration of the hydrogen peroxide vapour in the sampling bag was measured using the gas detector tube simultaneously. In the experiments of lactic acid or choline vapour measurement, the concentration of lactic acid or choline solution was regulated so that the gaseous concentration after vaporization would be equivalent to an examination value. The lactic acid or choline solution was dropped into a test tube and then the test tube was sealed. After the sample solution was completely vaporized, the sensitive area of the biosniffer was put into the test tube and the sensor output was monitored. Then, to evaluate the crosstalk of these three types of biosniffer for digital chemocode scanning, odourless chemicals such as 2 ppm hydrogen peroxide, $10 \mathrm{ppm}$ lactic acid, and $100 \mathrm{ppm}$ choline vapour were applied to these biosniffers and the changes in their output currents were assessed.

\subsection{Experimental setup for digital chemocode scanning}

The biosniffers were used for scanning 3 bits of the digital chemocode constructed using hydrogen peroxide, lactic acid, and choline vapours. Figure 2 shows an experimental setup for scanning the digital chemocode with the biosniffers. The concentrations of hydrogen peroxide, lactic acid, and choline solution were regulated so that the gaseous concentrations after vaporization would be 8.0, 10.0, and 1000 ppm, respectively. $20 \mu \mathrm{l}$ of each solution was dropped to a filter paper as 3 bits of chemocode with a micropipette. The filter paper marked with the odourless chemical code was placed in a screw bottle. After drying for $2 \mathrm{~h}$ at $50^{\circ} \mathrm{C}$, the sensing tips of the three biosniffers were placed in a screw bottle and the concentrations of the vaporized odourless chemicals were measured. 


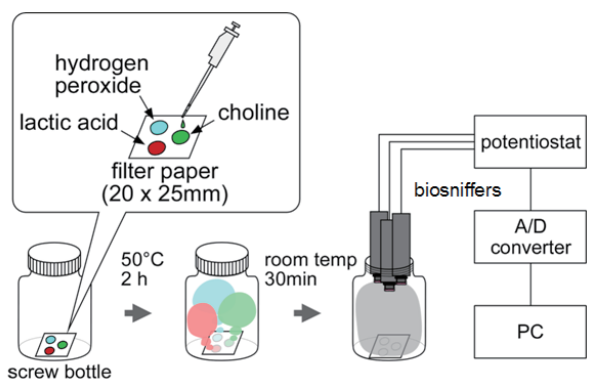

Fig. 2. (Color online) Experimental setup for odourless watermark scanning with biosniffers.

\section{Results and Discussion}

\subsection{Characteristics of biosniffer in gas phase}

Figure 3 shows the typical responses of the LOD-immobilized biosniffer that was monitored in the test tube filled with gaseous lactic acid. The $y$-axis shows the changes from the initial output current measured in air. After the sensor output became stable in air, the biosniffer was placed in the test tube at the point shown by the arrow on the graph. The change in output current of the biosniffer was increased by the enzyme reaction with volatile substrate at the enzyme membrane. The response time to reach $90 \%$ of the steady-state current after applying the lactic acid vapour was approximately $44 \mathrm{~s}$ at $1 \mathrm{ppm}$. Similar behaviours were obtained from the results of the examination of the biosniffers for hydrogen peroxide and choline vapours with the response time of approximately $1 \mathrm{~min}$. After applying the gaseous substrate, the sensor output showed the change induced by the enzymatic reaction for each biosniffer.

Figure 4 shows the calibration plot of these biosniffers against hydrogen peroxide, lactic acid, and choline vapours. Each biosniffer showed a linear response to the gaseous concentration of the enzyme reaction substrate. The catalase-, LOD-, and CODimmobilized biosniffers were calibrated against hydrogen peroxide from 0.4 to 12.5 ppm, lactic acid from 0.01 to $10.0 \mathrm{ppm}$, and choline vapours from 1.0 to $1000 \mathrm{ppm}$, respectively. The calibration curve of the catalase-immobilized biosniffer was shown as the following eq. (4) with the correlation coefficient of 0.996 deduced from the regression analysis of the linear plots by a least-squares method.

$$
\text { Sensor output }(\mu \mathrm{A})=0.010+0.253[\text { hydrogen peroxide }(\mathrm{ppm})]
$$

The calibration range of this biosniffer for hydrogen peroxide covers the threshold limit value $(1 \mathrm{ppm})$ of ACGIH. Similarly, the calibration curves of the LOD- and CODimmobilized biosniffer were shown as eqs. (5) and (6) deduced from the logarithmic regression analysis of the semilog plot by a least-squares method with correlation coefficients of 0.975 and 0.956 , respectively. 

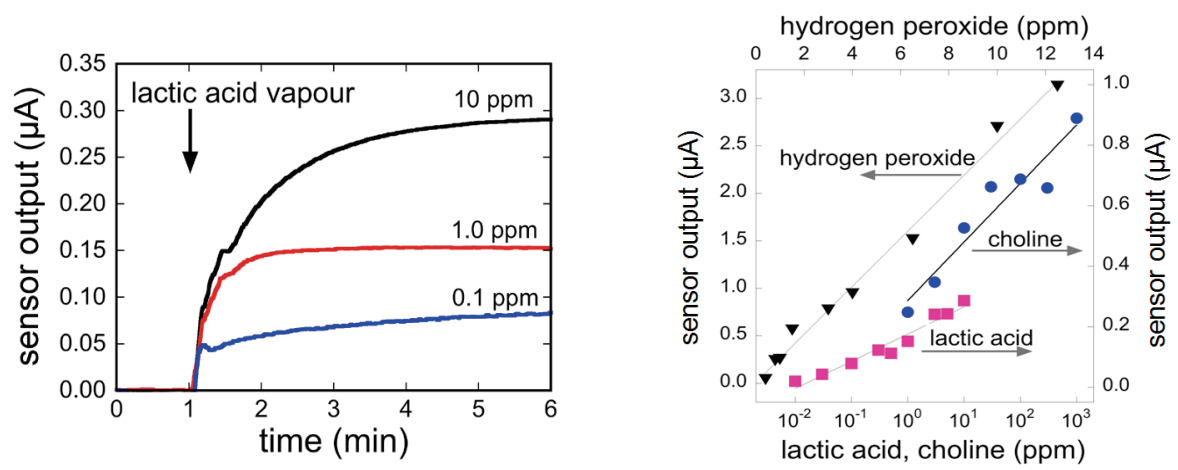

Fig. 3 (left). (Color online) Typical responses of biosniffer for lactic acid vapour. The lactic acid vapour was applied at the points shown by the arrows on the graph.

Fig. 4 (right). (Color online) Calibration curve of biosniffers for hydrogen peroxide, lactic acid, and choline vapours.

$$
\begin{aligned}
& \text { Sensor output }(\mu \mathrm{A})=0.176+0.090 \log ([\operatorname{lactic} \operatorname{acid}(\mathrm{ppm})]) \\
& \text { Sensor output }(\mu \mathrm{A})=0.286+0.194 \log ([\text { choline }(\mathrm{ppm})])
\end{aligned}
$$

In experiments involving liquid-phase measurements of hydrogen oxide, lactic acid, and choline by using the enzyme-immobilized biosensors, good reproducibility of coefficients of variance of $5.59,1.19$, and $5.96 \%(n: 20)$ were obtained from over $2 \mathrm{~h}$ of experiment, respectively. Also, in the gas-phase experiments, the three types of biosniffer maintained their performance throughout more than 15 times and $2 \mathrm{~h}$ of experiments. However, the performance of the biosniffer would decrease owing to decreasing dissolved oxygen concentration, accumulation of enzyme reaction products, and enzyme deactivation. In these cases, a flow cell system for circulating buffer solution to rinse and clean the sensing area of the biosniffer should be effective. ${ }^{(44)}$ A biosniffer for gaseous methyl mercaptan with a flow cell system could be used for a halfday-long measurement. ${ }^{(46,47)}$

To evaluate the selectivity of the three types of biosniffer, we applied each odourless chemical vapour such as hydrogen peroxide, lactic acid, and choline to each biosniffer. Figure 5 shows the sensor outputs to $2 \mathrm{ppm}$ hydrogen peroxide, $10 \mathrm{ppm}$ lactic acid, and $100 \mathrm{ppm}$ choline vapours. The sensor outputs were converted into relative values (\%) against the output current obtained from the specific reaction of the enzyme to the target odourless vapour for each biosniffer. Selectivity is the major characteristic of the biosensor/biochemical gas sensor, which was described previously.(22-24,42,43,47) Also, each biosniffer for gaseous hydrogen peroxide, lactic acid, and choline showed high selectivity against the three types of odourless chemical vapour for the 3-bit digital chemocodes. Thus, the crosstalk between the applied odourless vapours was not obtained from the 


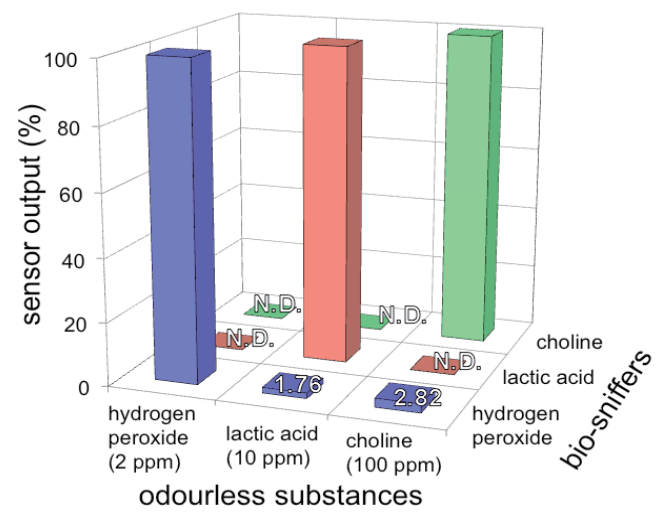

Fig. 5. (Color online) Odourless and colourless chemocode recognition by biochemical sniff scanner.

biosniffer outputs. These results indicate that the information on the presence/absence of odourless and colourless volatile chemicals could be available as a digital code and scanned with the biosniffers. The selectivity of the gas sensor should be evaluated for gas components included in the usage environment. In this study, biosniffers for the odourless watermark system were evaluated on the assumption of usage in a living atmosphere. When the odourless watermark system would be used in other environments such as an industrial plant, the selectivity of the biosniffers for gaseous components in the atmosphere should be evaluated. Generally, the selectivity of the biosensor is caused by the enzyme specificity. Therefore, it seemed that the biosniffers would be available to develop the odourless watermark system with volatile chemocode.

\subsection{Digital chemocode scanning using biosniffers}

The biosniffers were used for scanning 3 bits of the digital chemocode constructed by using hydrogen peroxide, lactic acid, and choline vapours. Figure 6 shows the setup of the 3-bit odourless chemical codes, typical examples of the biosniffer output, and the results of digital pattern recognition. The scanning of the digital chemocode was started at the point of $1 \mathrm{~min}$ on the graph. The detection thresholds of the odourless vapours were defined as $0.5 \mu \mathrm{A}$ for hydrogen peroxide and choline vapours, and $0.1 \mu \mathrm{A}$ for lactate vapour at the point of $1 \mathrm{~min}$ after the start the measurement. The three types of biosniffer successfully recognized eight patterns of the odourless chemical code.

The mechanism of the enzyme-based biosniffers in this study is different from that of our olfactory system where receptor proteins combine with chemicals. Therefore biosniffers for other odourless substances can be constructed. By using more pairs of odourless substances and biosniffers with high selectivity, a long bit of digital chemocode should be achieved. 


\begin{tabular}{|c|c|c|c|c|c|c|}
\hline \multirow{2}{*}{ odorless substrate } & \multicolumn{6}{|c|}{ digital code } \\
\hline & A B & C D & $E$ & $F$ & G & $\mathrm{H}$ \\
\hline hydrogen peroxide & 00 & 00 & $\mathrm{x}$ & $\mathrm{x}$ & $\mathrm{x}$ & $\mathrm{x}$ \\
\hline lactic acid & 00 & $x \times$ & 0 & 0 & $x$ & $\mathrm{x}$ \\
\hline choline & $0 x$ & $0 x$ & 0 & $\mathrm{x}$ & 0 & $\mathrm{X}$ \\
\hline
\end{tabular}

Typical examples of biosniffer output
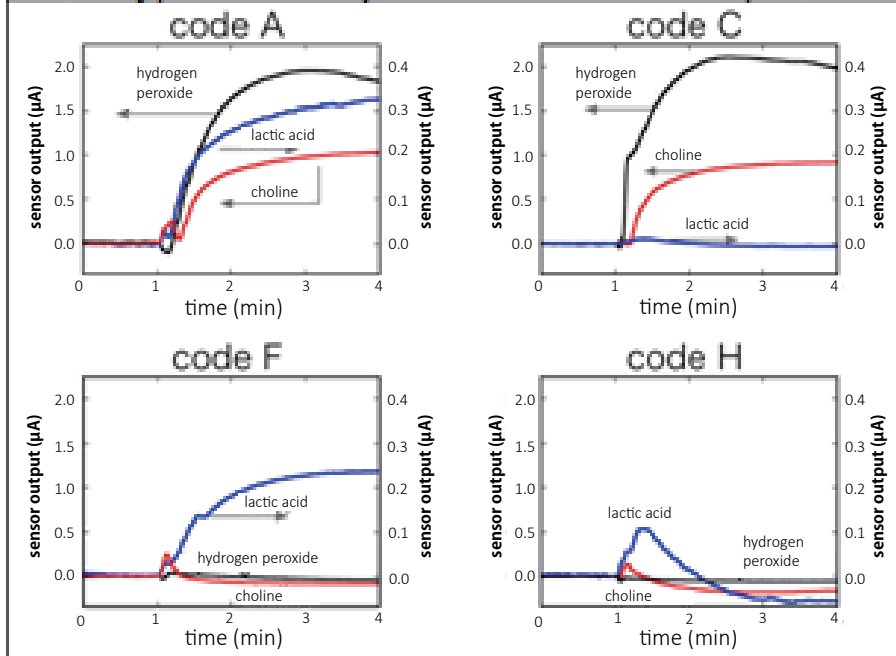

pattern of biosniffer response

\begin{tabular}{lllllllll}
\multirow{2}{*}{ biosniffer } & \multicolumn{6}{c}{ digital pattern } \\
\cline { 2 - 8 } & A & $B$ & $C$ & $D$ & $E$ & $F$ & $G$ & $H$ \\
\hline hydrogen peroxide & $O$ & $O$ & $O$ & $O$ & $X$ & $X$ & $X$ & $X$ \\
lactic acid & $O$ & $O$ & $X$ & $X$ & $O$ & $O$ & $X$ & $X$ \\
choline & $O$ & $X$ & $O$ & $X$ & $O$ & $X$ & $O$ & $X$ \\
\hline & $O$ & : responded & & & \\
& $X$ & : no response
\end{tabular}

Fig. 6. (Color online) Odourless digital chemocodes recognized using biochemical sniff scanner. 


\section{Conclusions}

An odourless watermark system with biosniffers for hydrogen peroxide, lactic acid, and choline was developed. Each biosniffer consisted of the Clark-type dissolved oxygen electrode and the catalase/LOD/COD-immobilized membrane. The calibration property and selectivity of the biosniffers for gaseous phase were evaluated. Each biosniffer showed a linear response to the gaseous concentration of the enzyme reaction substrate. The calibration ranges of each biosniffer for hydrogen peroxide, lactic acid, and choline vapours were from 0.4 to $12.5,0.01$ to 10.0 , and 1.0 to $1000 \mathrm{ppm}$ with correlation coefficients of $0.996,0.975$, and 0.956 , respectively. Then, these biosniffers were applied for scanning 3 bits of the digital chemocode constructed using hydrogen peroxide, lactic acid, and choline vapours. The three types of biosniffer successfully recognized eight patterns of printed odourless chemical code.

\section{Acknowledgements}

This study was partially supported by the Japan Society for the Promotion of Science (JSPS) Grants-in-Aid for Scientific Research and the Ministry of Education, Culture, Sports, Science and Technology (MEXT) Special Funds for Education and Research "Advanced Research Program in Sensing Biology".

\section{References}

1 Y.-G. Fu and R.-M. Shen: Comput. Stand. Interfaces 30 (2008) 115.

2 H. Qi, D. Zheng and J. Zhao: Signal Process. 88 (2008) 174.

3 L. O'Gorman: Information Secur. Tech. Rep. 3 (1998) 21.

4 X. Wu, D. Zhang, K. Wang and B. Huang: Pattern Recognit. 37(2004) 1987.

5 L. Wang, G. Leedham and D. S.-Y. Cho: Pattern Recognit. 41 (2008) 920.

6 B. E. Watt, A. T. Proudfoot and J. A. Vale: Toxicol. Rev. 23 (2004) 51.

7 L. J. Walsh: Aust. Dent. J. 45 (2000) 257.

8 P. P. Rosias, E. Dompeling, H. J. Hendriks, J. W. Heijnens, R. A. Donckerwolcke and Q. Jöbsis: Pediatr. Allergy Immunol. 15 (2004) 4.

9 P. Montuschi: Nat. Rev. Drug Discov. 1 (2002) 238.

10 P. Montuschi and P. J. Barnes: Trends Pharmacol. Sci. 23 (2002) 232.

11 S. A. Kharitonov and P. J. Barnes: Biomarkers 7 (2002) 1.

12 A. Emelyanov, G. Fedoseev, A. Abulimity, K. Rudinski, A. Fedoulov, A. Karabanov and P. J. Barnes: Chest 120 (2001) 1136.

13 I. Horvath, L. E. Donnelly, A. Kiss, S. A. Kharitonov, S. Lim, K. F. Chung, and P. J. Barnes: Am. J. Respir. Crit. Care Med. 158 (1998) 1042.

14 American Conference of Governmental Industrial Hygienists (ACGIH): (Cincinnati, 1994) p. 23.

15 S. Consolo, C. F. Wu, F. Fiorentini, H. Ladinsky and A. Vezzani: J. Neurochem. 48 (1987) 1459.

16 Q. Xin and R. M. Wightman: Brain Res. 776 (1997) 126. 
17 Occupational Safety and Health Administration (OSHA): Hydrogen Peroxide in Workplace Atmospheres (Method no.: ID-126-SG, Control no.: T-ID126SG-PV-01-0201-M) https:// www.osha.gov/dts/sltc/methods/partial/t-id126sg-pv-01-0201-m/t-id126sg-pv-01-0201-m. html.

18 I. Taizo, A. Sinichi and K. Kawamura: PDA J. Pharm. Sci. Technol. 52 (1998) 13.

19 R. Toniolo, P. Geatti, G. Bontempelli and G. Schiavon: J. Electroanal. Chem. 514 (2001) 123.

20 H. L. Huang, P. K. Dasgupta, Z. Genfa and J. Wang: Anal. Chem. 68 (1996) 2062.

21 K. Mitsubayashi, H. Matsunaga, G. Nishio, S. Toda and Y. Nakanishi: Biosens. Bioelectron. 20 (2005) 1573.

22 H. Saito, Y. Kaneko, Y. Hashimoto, T. Shirai, H. Kudo, K. Otsuka and K. Mitsubayashi: Sens. Actuators, B 123 (2007) 877.

23 K. Mitsubayashi, H. Matsunaga, G. Nishio, S. Toda, Y. Nakanishi, H. Saito, M. Ogawa and K. Otsuka: Sens. Actuators, B 108 (2005) 660.

24 K. Mitsubayashi, Y. Kubotera, K. Yano, Y. Hashimoto, T. Kon, S. Nakakura, Y. Nishi and H. Endo: Sens. Actuators, B 103 (2004) 463.

25 X. J. Liu, Y. Xu, X. Ma and G. X. Li: Sens. Actuators, B 106 (2005) 284.

26 H. Zhou, X. Gan, J. Wang, X. L. Zhu and G. X. Li: Anal. Chem. 77 (2005) 6102.

27 X. Gan, T. Liu, J. Zhong, X. J. Liu and G. X. Li: Chembiochem. 5 (2004) 1686.

28 Y. Xu, W. L. Peng, X. J. Liu and G. X. Li: Biosens. Bioelectron., 20 (2004) 533.

29 C. Xiaoqiang, M. L. Chang, Z. Jianfeng and Y. Shucong: Biosens. Bioelectron. 22 (2007) 3288.

30 K. Wang, J.-J. Xu and H.-Y. Chen: Sens. Actuators, B 114 (2006) 1052.

31 C. A. Marquette and L. J. Blum: Sens. Actuators, B 90 (2003) 112.

32 C. A. Marquette, A. Degiuli and L. J. Blum: Biosens. Bioelectron. 19 (2003) 433.

33 S. Suman, R. Singhal, A. L. Sharma, B. D. Malthotra and C. S. Pundir: Sens. Actuators, B 107 (2005) 768.

34 F. R. Alexander, S. Kaushik, S. Aleksandr and V. P. Michael: Sens. Actuators, B 81 (2002) 359.

35 J. J. Mitala Jr. and A. C. Michael: Anal. Chim. Acta. 556 (2006) 326.

36 Z. Song, J. Huang, B. Wu, H. Shi, J. Anzai and Q. Chen: Sens. Actuators, B 115 (2006) 626.

37 F. Qu, M. Yang, J. Jiang, G. Shen and R. Yu: Anal. Biochem. 344 (2005) 108.

38 M. Yang, Y. Yang, Y. Yang, G. Shen and R. Yu: Anal. Chim. Acta. 530 (2005) 205.

39 O. N. Schuvailo, S. V. Dzyadevych, A. V. El'skaya, S. Gautier-Sauvigné, E. Csöregi, R. Cespuglio and A. P. Soldatkin: Biosens. Bioelectron. 21 (2005) 87.

40 A. Guerrieri, V. Lattanzio, F. Palmisano and P. G. Zambonin: Biosens. Bioelectron. 21 (2006) 1710 .

41 K. Kudo, T. Goto, T. Saito, H. Saito, K. Otsuka and K. Mitsubayashi: Microchim. Acta. 160 (2008) 3421.

42 H. Saito, Y. Kaneko, Y. Hashimoto, T. Shirai and K. Mitsubayashi: Int. J. Environ. Anal. Chem. 86 (2006) 1057.

43 K. Otsuka, T. Goto, H. Amagai, N. Shii, H. Endo and K. Mitsubayashi: Int. J. Anal. Chem. 86 (2006) 1049.

44 T. Minamide, K. Mitsubayashi, N. Jaffrezic-Renault, K. Hibi, H. Endo and H. Saito: Analyst 130 (2005) 1490.

45 K. Ichimura: J. Polym. Sci. 22 (1984) 2817.

46 K. Mitsubayashi, T. Minamide, K. Otsuka, H. Kudo and H. Saito: Anal. Chim. Acta. 573-574 (2006) 75.

47 T. Minamide, K. Mitsubayashi and H. Saito: Sens. Actuators, B 108 (2005) 639. 